\title{
Efeito de diferentes doses de ácido retinoico sobre a resistência óssea de ratos jovens
}

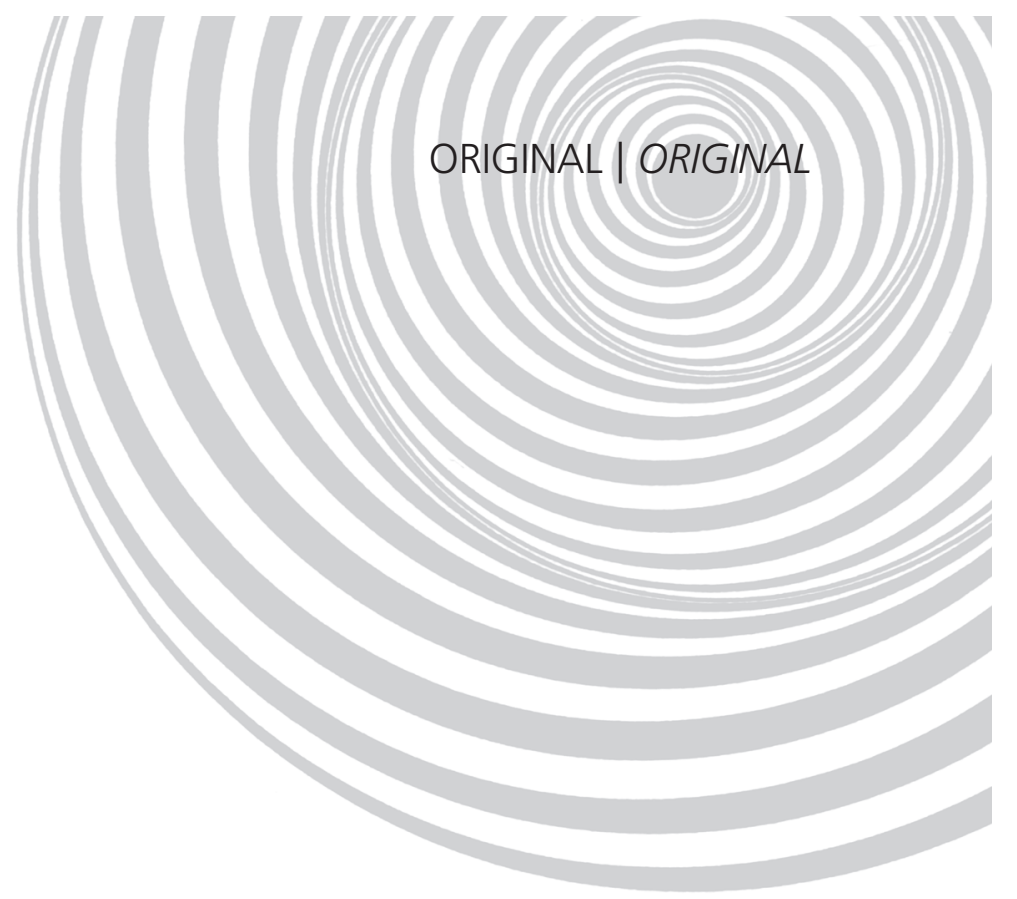

\author{
Effect of varied doses of retinoic acid \\ on young rats' bone resistance
}

Luciana Bronzi de SOUZA'

Cristiana Maria Murbach FREIRE ${ }^{2}$

Renata Noêmia Alves de ALMEIDA ${ }^{3}$

Sérgio Swain MÜLLER ${ }^{4}$

Sérgio Alberto Rupp PAIVA ${ }^{5}$

Gláucia Maria Ferreira da Silva MAZETO ${ }^{6}$

RE S U M O

\section{Objetivo}

Avaliar os efeitos da suplementação de diferentes doses de todo-trans ácido retinóico sobre a resistência óssea, por meio de ensaio biomecânico de flexão, em tíbia de ratos jovens.

\section{Métodos}

Foram estudados 58 ratos jovens, com quatro diferentes doses de vitamina A em suas dietas, sendo divididos em 4 grupos: grupo-controle $(n=15)$, sem acréscimo de todo-trans ácido retinoico; grupo com acréscimo de 0,3mg de todo-trans ácido retinoico por kg de ração $(n=13)$; grupo com 10mg de todo-trans ácido retinoico por kg de ração $(n=15)$; e grupo com 50mg de todo-trans ácido retinoico por kg de ração $(n=15)$. O estudo durou 30 dias. Após o sacrifício dos animais, suas patas esquerdas foram congeladas, dissecadas e as tíbias submetidas ao ensaio de flexão. Foram avaliados a carga máxima e o coeficiente de rigidez. Foi aplicada análise de variância one-way. O nível de significância estatístico adotado foi $p<0,05$.

\section{Resultados}

Os valores médios de carga máxima (em Newton) foram: grupo-controle =37,94, DP=4,76; grupo todo-trans ácido retinoico 0,3=36,49, DP=4,38; grupo todo-trans ácido retinoico 10=40,12, DP=6,03; grupo todo-trans

1 Universidade Estadual Paulista Júlio de Mesquita Filho, Faculdade de Medicina de Botucatu, Departamento de Saúde Pública. Botucatu, SP, Brasil.

2 Universidade Estadual Paulista Júlio de Mesquita Filho, Faculdade de Medicina de Botucatu, Departamento de Clínica Médica. Rubião Júnior s/n., 18618-000, Botucatu, SP, Brasil. Correspondência para/Correspondence to: G.M.F.S. MAZETO. E-mail: <gmazeto@fmb.unesp.br>.

${ }^{3}$ Nutricionista. Botucatu, SP, Brasil.

${ }^{4}$ Universidade Estadual Paulista Júlio de Mesquita Filho, Faculdade de Medicina de Botucatu, Departamento de Cirurgia e Ortopedia. Botucatu, SP, Brasil. 
376 | L.B. SOUZA et al.

ácido retinoico 50=35,68, $\mathrm{DP}=5,22(p=0,107)$. Os valores médios de coeficiente de rigidez (em Newton/milímetros) foram: grupo-controle $=31,84 \mathrm{DP}=6,75$; grupo todo-trans ácido retinoico 0,3=29,18, $\mathrm{DP}=4,35$; grupo todo-trans ácido retinoico 10=35,48, DP=8,14; grupo todo-trans ácido retinoico 50=30,31, DP=7,14 $(p=0,85)$.

\section{Conclusão}

Conclui-se que a exposição a diferentes doses de todo-trans ácido retinoico, em ratos, durante 30 dias, não exerce efeito sobre a resistência óssea, quando avaliada por ensaios biomecânicos.

Termos de indexação: Biomecânica. Osteoporose. Ratos. Tretinoína.

\section{A B S T R A C T}

\section{Objective}

This study assessed the effects of different doses of all-trans retinoic acid on bone resistance by conducting a biomechanical flexion study on young rats' tibias.

\section{Methods}

Fifty-eight young rats were divided into four groups according to the all-trans retinoic acid content of their diets: control group $(n=15)$, chow not enriched with all-trans retinoic acid; chow enriched with $0.3 \mathrm{mg}$ of all-trans retinoic acid per kilogram ( $n=13)$; chow enriched with $10 \mathrm{mg}$ of all-trans retinoic acid per kilogram $(n=15) ;$ and chow enriched with 50mg of all-trans retinoic acid per kilogram (n-15). After 30 days of this diet, the animals were killed, their left paws were frozen and dissected and the tibias were submitted to the flexion study which assessed maximum force and shear modulus. One-way analysis of variance was used with significance set at $p<0.05$.

\section{Results}

The mean maximum force values in newtons (SD) were: control group $=37.94, S D=4.76 ; 0.3 \mathrm{mg}$ group = 36.49, $S D=4.38 ; 10 \mathrm{mg}$ group $=40.12, S D=6.03 ; 50 \mathrm{mg}$ group $=35.68, S D=5.22(p=0.107)$. The mean shear modulus values $(S D)$ in newtons/millimeter were: control group $=31.84, S D=6.75 ; 0.3 \mathrm{mg}$ group $=29.18, S D=4.35$; $10 \mathrm{mg}$ group $=35.48, S D=8.14 ; 50 \mathrm{mg}$ group $=30.31, S D=7.14(p=0.85)$.

\section{Conclusion}

Biomechanical studies showed that different doses of all-trans retinoic acid for 30 days had no effect on young rats' bone resistance.

Indexing terms: Biomechanics. Osteoporosis. Rats. Tretinoin.

\section{N T R O D U Ç Ã O}

A vitamina A pré-formada (todo-trans-retinol e seus ésteres) e a pró-vitamina $A$ (beta-caroteno) são nutrientes dietéticos essenciais, metabolizados em retinol no organismo. 0 retinol é oxidado a retinal, que é essencial para a visão (principalmente noturna), e ácido retinoico, um ligante de fator de transcrição, com importantes funções na regulação de genes envolvidos na morfogênese, diferenciação e proliferação celulares ${ }^{1}$.

A deficiência de vitamina A caracteriza-se por xeroftalmia, cegueira noturna e aumento da suscetibilidade a doenças ${ }^{2}$. Ainda ocorre em alguns países, tendo sido relatada em várias regiões do Brasil, com alta prevalência em diferentes faixas etárias ${ }^{3}$. A suplementação do micronutriente, em suas diferentes formas, a populações com insuficiência do mesmo, tem sido associada a muitos benefícios, entre os quais a redução da mortalidade em geral e por câncer, particularmente o de origem gástrica ${ }^{4}$.

Além dos efeitos obtidos com a reposição vitamínica, outros resultados terapêuticos da vitamina A são relatados. A isotretinoína (ácido retinoico 13-cis), o mais potente inibidor conhecido da produção sebácea, apresenta sucesso compro- 
vado no tratamento da acne, sendo amplamente utilizada ${ }^{5}$.

Assim, de forma geral, a prescrição de vitamina $A$, como suplemento ou terapia, parece encorajadora. Contudo, essa suplementação deve ser conduzida com cautela, uma vez que o excesso da vitamina também pode ser tóxico ao organismo, resultando em hepatotoxicidade, alterações visuais e anormalidades craniofaciais em fetos $^{2}$. Além disso, em modelos animais, a suplementação de retinol tem sido associada a dano de DNA e rearranjos cromossômicos ${ }^{6}$.

Também têm sido referidos efeitos deletérios do excesso de retinol sobre o tecido ósseo ${ }^{7}$, tais como a estimulação da reabsorção e a inibição da formação, resultando em perda óssea ${ }^{8}$. 0 Todo-Trans Ácido Retinoico (ATRA) parece regular a expressão de diversas proteínas nos osteoblastos e osteoclastos, incluindo proteínas matrizes e enzimas lisossomais, que podem desempenhar um papel direto na reabsorção óssea ${ }^{9}$. A constante perda óssea pode culminar no aparecimento da osteoporose $^{8}$.

A osteoporose constitui um problema de saúde pública, na medida em que apresenta elevada prevalência e acarreta fraturas que prejudicam a qualidade de vida e aumentam a mortalidade. Na América Latina, estima-se que ela acometa de $12 \%$ a $22 \%$ das mulheres com 50 anos ou mais de idade ${ }^{10}$. Incide, particularmente, em pacientes do sexo feminino e apresenta relação causal com o envelhecimento e com a deprivação hormonal que ocorre após a menopausa, entre outros fatores ${ }^{11}$. Assim, esse grupo populacional é mais vulnerável aos demais agressores da saúde óssea. Com relação ao uso dos derivados da vitamina $\mathrm{A}$, tem sido relatado aumento das taxas de fraturas de quadril em mulheres ${ }^{12}$, particularmente no período pós-menopausa ${ }^{13}$, com elevada ingestão de retinol, tanto por dieta como por suplementação.

O estudo da resistência biomecânica óssea vem sendo utilizado para análise da qualidade de ossos suscetíveis a fraturas. Com relação à avaliação do efeito dos compostos derivados da vitamina A, por exemplo, estudo utilizando o ensaio mecânico de flexão, em ossos de ratas maduras, alimentadas com elevadas doses de ácido retinoico em suas dietas, por 12 semanas, demonstrou ser necessário menor força Newton (N) para romper ossos dos animais que receberam maior dose, indicando efeitos negativos do excesso da ingestão de vitamina A sobre a resistência óssea ${ }^{14}$.

Considerando-se a elevada prevalência da osteoporose, suas implicações para a saúde pública e a crescente prescrição de vitamina A e seus compostos, torna-se importante a avaliação dos efeitos esqueléticos das diversas doses dessa vitamina. Uma vez que os ensaios de flexão constituem métodos aplicáveis de avaliação da resistência óssea, ensaios com modelos diferentes devem ser implementados, para um maior conhecimento desta, frente ao uso de compostos derivados da vitamina A.

Assim, o objetivo deste estudo foi avaliar os efeitos da suplementação com diferentes doses de ATRA sobre a resistência óssea, por meio de ensaio biomecânico de flexão, em tíbia de ratos jovens.

\section{MÉ T O D O S}

Foram estudados 58 ratos Wistar (Rattus novergicus, var. albinus), machos, filhotes, com aproximadamente 1 mês de vida. Os animais foram acomodados, por um período de 30 dias, à temperatura de $25 \pm 1^{\circ} \mathrm{C}$, com período claro/escuro de 12 horas. Foram alojados cinco animais por caixa de polietileno, com livre acesso a água e ração. A dieta utilizada foi AIN-93G, por ser preparada com quantidade mínima de vitamina A (4000 UI), além de ser quimicamente definida e adequada ao crescimento de roedores ${ }^{15}$. O estudo foi conduzido com rígido cumprimento dos guias da Instituição e dos Conselhos Nacionais de Pesquisa sobre uso e cuidado de animais de laboratório, além de atender à legislação específica do país, tendo sido aprovado pela Comissão 
de Ética em Experimentação Animal da Instituição em que foi realizado o experimento.

Os animais foram divididos em quatro grupos, cada um dos quais recebeu dietas adicionadas de diferentes doses de ATRA: de Grupo-Controle (C) $(n=15)$ : sem adição de ATRA; grupo ATRA $0,3(n=15)$ : adição de $0,3 \mathrm{mg}$ de ATRA por $\mathrm{kg}$ de dieta (1.000 UI, equivalente a quantidade basal); grupo ATRA 10 ( $n=13)$ : adição de $10 \mathrm{mg}$ de ATRA por $\mathrm{kg}$ de dieta (33.333 UI, equivalente à primeira dose farmacológica); grupo ATRA $50(n=15)$ : adição de 50mg de ATRA por kg de dieta (166.667 $\mathrm{UI}$, equivalente à segunda dose farmacológica).

As medidas de peso corporal e ingestão alimentar foram aferidas semanalmente. Para verificação da ingestão alimentar foram utilizados 20 ratos, divididos em quatro caixas, uma de cada tratamento. A dieta foi pesada e oferecida aos animais. Após 24 horas, a dieta que restou foi novamente pesada. A diferença entre o peso da dieta oferecida e o peso da dieta restante, em 24 horas, foi dividida pelo número de ratos por caixa e por dia. A partir desse valor da ingestão alimentar, foi calculada a ingestão de ATRA/dia.

Ao fim de quatro semanas, os ratos foram anestesiados com cloridrato de cetamina $(50 \mathrm{mg} / \mathrm{kg})$ e cloridrato de xilidino $(1 \mathrm{mg} / \mathrm{kg})$, via intramuscular. A mistura desses analgésicos causa taquicardia no roedor, levando-o à morte, sem efeitos diretos sobre o estudo.

As patas traseiras esquerdas dos animais foram retiradas e acondicionadas em um freezer a uma temperatura média de $-40^{\circ} \mathrm{C}$, até o momento da análise. No momento de ensaio biomecânico, as patas foram descongeladas à temperatura ambiente e posteriormente dissecadas, sendo então efetuado o ensaio ${ }^{16}$.

Foram realizados ensaios de flexão de tíbia. Estas foram acondicionadas em uma morsa aberta, a uma distância de 2/3 do tamanho do osso, exercendo-se a flexão até a ruptura do mesmo. Para tal ensaio, foi utilizada a Máquina Universal de Ensaios Mecânicos EMIC (Equipamentos e Sistemas de Ensaio Ltda (Curitiba - PR), modelo DL
10000. A precisão do sistema é de $(0,018+F /$ 3700) KN, apurada dentro das especificações das normas ABTN, NBR6156 e NBR6674. A aferição é realizada periodicamente pelo fabricante. $\mathrm{O}$ aparelho operou conjuntamente com microcomputador sob o sistema operacional Windows 2000, sendo utilizado o programa de informática Mtest versão 1.0. Esse programa gera um gráfico força $x$ deformação, a partir do qual puderam-se calcular as variáveis analisadas.

Foram avaliados a Carga Máxima (N), definida como a carga suportada pelo corpo de prova até a ruptura, bem como o Coeficiente de rigidez (K) (N/mm), definido como a relação entre carga e deformação no trecho reto de curva até o limite de elasticidade, sendo, portanto indicativo da rigidez da estrutura.

A padronização do processo deu-se pelo mesmo espaçamento da morsa em todos os ensaios, e pela colocação dos ossos em uma mesma posição ${ }^{16}$.

Os resultados estão demonstrados em Mé$\operatorname{dias}(\mathrm{M})$, Desvio-Padrão (DP). O software Statistica ${ }^{17}$ versão 6.0 foi utilizado para a realização das análises. Foi aplicada análise de variância one-way (ANOVA), seguida do teste de comparações múltiplas de Tukey, para verificar a diferença entre os tratamentos. O nível de significância estatístico adotado foi $p<0,05$.

\section{RESULTA D OS}

Ao final da suplementação com ATRA, o peso corporal dos ratos foi semelhante nos diferentes grupos $(p=0,178)$, como pode ser observado na Figura 1.

A ingestão da dieta foi calculada por rato e apresentada por valor diário (C: 16g; ATRA 0,3: 18g; ATRA 10: 16g; ATRA 50: 18g). Em relação à quantidade de ATRA ingerida por dia, o grupo controle nada ingeriu; o grupo ATRA 0,3 ingeriu 0,006mg (20 UI); o grupo ATRA 10 ingeriu $0,17 \mathrm{mg}$ (567 UI); e o grupo ATRA 50 ingeriu 0,92 mg (3.067 UI). 
Os valores médios de carga máxima e do coeficiente de rigidez (K) dos quatro grupos não foram estatisticamente diferentes (Tabela 1).

\section{I S C U S S Ã O}

O exato mecanismo pelo qual a vitamina A pode influenciar a saúde do esqueleto não é claro 9,18,19. Contudo, historicamente, estudos em humanos ${ }^{20-22}$ e animais ${ }^{23-25}$ têm relatado que a toxicidade por essa vitamina aumenta a reabsorção e diminui a formação óssea.

Embora o elevado consumo de vitamina A possa contribuir para o desenvolvimento de

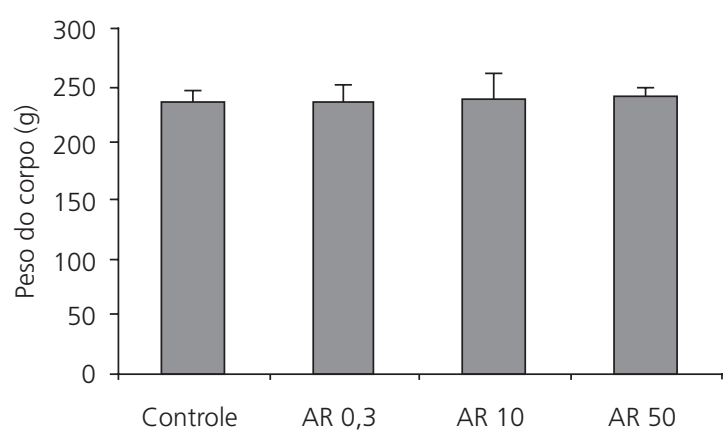

Figura 1. Valores médios (desvio-padrão) do peso corporal dos ratos dos grupos controle (sem adição de todo-trans ácido retinoico na dieta); AR 0,3 (ATRA 0,3; adição de 0,3mg de ATRA por kg de dieta); AR 10 (ATRA 10; adição de 10mg de todo-trans ácido retinoico por kg de dieta) e AR 50 (ATRA 50; adição de 50mg de todo-trans ácido retinoico por $\mathrm{kg}$ de dieta). ANOVA de 1 via, $p=0,178$. osteoporose, não está claro qual a quantidade necessária para causar toxicidade esquelética.

O presente estudo demonstrou que diferentes concentrações de ácido retinoico, pelo período de um mês, não exerceram influência sobre a resistência óssea em ratos jovens, diferentemente do descrito por Johansson et al. ${ }^{14}$, que observaram ser necessário menor quantidade de força, em N, para romper ossos de ratos que receberam maior quantidade de ácido retinoico em sua dieta. Contudo, devem-se ressaltar as diferenças existentes entre os estudos, tais como o sexo e a idade dos animais. Johansson et al..$^{14}$ utilizaram fêmeas maduras, enquanto o presente estudo avaliou ratos jovens. Em humanos, por exemplo, as mulheres apresentam menor massa óssea, sendo que esta evolui de forma crescente até determinada faixa etária, quando começa o seu declínio. $A$ ação dos compostos ligados à vitamina $A$ sobre o pico de massa óssea humana, e talvez sobre a resistência do tecido ósseo, é discutível, sendo que Hogstrom et al. ${ }^{26}$ não encontraram associação entre este e os níveis séricos de retinol, em homens jovens.

Outro ponto discordante com o estudo de Johansson et al..$^{14}$ é o tempo de tratamento. No presente trabalho, devido ao curto período de administração da dieta enriquecida com ATRA, é pouco provável que este afetasse negativamente o esqueleto ${ }^{27}$, ao passo que Johansson et al. ${ }^{14}$ trataram os ratos por um período três vezes maior. Poder-se-ia inferir, a priori, que a exposição por mais tempo ao interferente ósseo deveria resultar

Tabela 1. Valores médios de carga máxima e coeficiente de rigidez (K), obtidos em ensaios biomecânicos de ratos submetidos a suplementação de diferentes dosagens de ATRA.

\begin{tabular}{|c|c|c|c|c|c|c|c|c|c|}
\hline \multirow{3}{*}{ Variáveis biomecânicas } & \multicolumn{8}{|c|}{ Grupos $^{*}$} & \multirow{3}{*}{$p$} \\
\hline & \multicolumn{2}{|c|}{ Controle } & \multicolumn{2}{|c|}{ ATRA 0,3 } & \multicolumn{2}{|c|}{ ATRA 10} & \multicolumn{2}{|c|}{ ATRA 50} & \\
\hline & M & DP & M & $\mathrm{DP}$ & M & $\mathrm{DP}$ & $M$ & DP & \\
\hline Carga máxima (N) & 37,94 & 4,76 & 36,49 & 4,38 & 40,12 & 6,03 & 35,68 & 5,22 & 0,107 \\
\hline $\mathrm{K}(\mathrm{N} / \mathrm{mm})$ & 31,84 & 6,75 & 29,18 & 4,35 & 35,48 & 8,14 & 30,31 & 7,14 & 0,85 \\
\hline
\end{tabular}

${ }^{*}$ Controle (C) ( $\left.n=15\right)$ : sem adição de todo-trans ácido retinoico; ATRA 0,3 ( $\left.n=15\right)$ : adição de 0,3mg de todo-trans ácido retinoico por kg de dieta; ATRA 10 ( $n=13$ ): adição de 10mg de todo-trans ácido retinoico por kg de dieta; ATRA 50 ( $n=15)$ : adição de $50 \mathrm{mg}$ de todo-trans ácido retinoico por kg de dieta.

M: média; DP: desvio-padrão; ATRA: todo-trans ácido retinoico. 
em maior perda óssea. Porém, Li et al. ${ }^{28}$ não encontraram efeitos negativos na remodelação óssea com doses diárias de 300 UI, por 14 meses, em ratos idosos. Novamente, as idades diferentes dos animais dificultam uma comparação precisa entre os grupos.

Ainda, as doses de compostos ligados à vitamina A utilizadas diferem nos estudos. Embora Johansson et al. ${ }^{14}$ tenham utilizado doses superiores (9 $000 \mathrm{UI} / \mathrm{dia}$ ) às do presente estudo, aqueles autores não descreveram as doses efetivamente ingeridas pelos animais. Na presente pesquisa, apesar das elevadas doses farmacológicas ofertadas, aquelas efetivamente ingeridas foram bastante inferiores às relatadas como tóxicas. Além disso, Nieman \& Obbink ${ }^{29}$ concluíram que a toxicidade crônica oral, em ratos adultos, começa com cerca de 25.000 UI/dia. Enfim, essas diferenças metodológicas constituem pontos críticos na avaliação dos diferentes trabalhos.

Embora não tenham sido demonstradas alterações significativas com relação às variáveis biomecânicas do osso, estudos histológicos são necessários para a complementação desta investigação. Esse tipo de avaliação permitiria a contagem de osteoblastos e osteoclastos e, ainda, a determinação do tipo de cartilagem mais presente, uma vez que a atividade osteoclástica é maior em animais intoxicados com a vitamina $A^{21}$. Nesses casos, Hough et al. ${ }^{30}$ observaram osteoporose severa, aumento da reabsorção óssea, osteoclastose, degeneração de cartilagem e escassez de osteoide.

\section{CONCLUSÃ O}

A partir dos resultados obtidos, é possível concluir que a exposição de ratos jovens a diferentes doses de ATRA, por um período de um mês, não exerce efeito na resistência óssea, quando avaliada por meio de ensaios biomecânicos. Estudos com maior período de exposição dos animais ao composto, e com diferentes grupos quanto ao sexo e faixa etária, são necessários para que se possa avaliar seu efeito em longo prazo na resistência óssea.

\section{A GRADECIMENTOS}

À equipe do Laboratório Experimental do Departamento de Cirurgia e Ortopedia da Faculdade de Medicina de Botucatu pela realização dos ensaios biomecânicos, e a Maíra Barreto Malta e ao Prof. Dr. José Eduardo Corrente pela realização da análise estatística.

\section{COLABORADORES}

L. B. SOUZA participou da coleta e da tabulação de dados, da revisão da literatura e da redação final. C.C.M. FREIRE e R.N.A. ALMEIDA participaram da coleta dos dados, do desenvolvimento do projeto inicial. S.S. MÜLLER analisou os resultados dos ensaios biomecânicos. S.A.R. PAIVA participou na concepção e no desenho do estudo. G.M.F.S. MAZETO participação na concepção, no desenho e estudo e na revisão final.

\section{REFERÊ NCIAS}

1. Dawson MI. The importance of vitamin $A$ in nutrition. Curr Pharm Des. 2000; 6(3):311-25.

2. Fairfield KM, Fletcher RH. Vitamins for chronic disease prevention in adults: scientific review. JAMA. 2002; 287(23):3116-26.

3. Ramalho RA, Flores $H$, Saunders $C$. Hypovitaminosis A in Brazil: a public health problem. Rev Panam Salud Publica. 2002; 12(2):117-22.

4. Qiao YL, Dawsey SM, Kamangar F, Fan JH, Abnet CC, Sun XD, et al. Total and cancer mortality after supplementation with vitamins and minerals: follow-up of the Linxian General Population Nutrition Intervention Trial. J Natl Cancer Inst. 2009; 101(7):507-18.

5. Ganceviciene R, Zouboulis CC. Isotretinoin: state of the art treatment for acne vulgaris. J Dtsch Dermatol Ges. 2010; 8 (Suppl 1):S47-59.

6. Klamt F, Dal-Pizzol F, Roehrs R, Oliveira RB, Dalmolin $R$, Henriques JA, et al. Genotoxicity, recombinogenicity and cellular preneoplasic transformation induced by vitamin A supplementation. Mutat Res. 2003; 539(1-2):117-25.

7. Wolbach SB. Vitamin A deficiency and excess in relation to skeletal growth. J Bone Joint Surg. 1947; 29:171-92.

8. Binkley N Krueger D. Hypervitaminosis A and bone. Nutr Rev. 2000; 58:138-44. 
9. Rhode CM, Deluca $\mathrm{H}$. Bone resorption activity of all-trans retinoic acid is independent of vitamin $D$ in rats. J Nutr. 2003; 133:777-83.

10. Morales-Torres J, Gutiérrez-Ureña S, Osteoporosis committee of Pan-American League of Associations for Rheumatology. The burden of osteoporosis in Latin America. Osteoporos Int. 2004; 15(8):625-32.

11. Lanzillotti HS, Lanzillotti RS, Trotte APR, Dias AS, Bornand B, Costa EAMM. Osteoporose em mulheres na pós-menopausa, cálcio dietético e outros fatores de risco. Rev Nutr. 2003; 16(2):181-93. doi: 10.1590/S1415-52732003000200005.

12. Melhus $H$, Michaelsson K, Kindmark A, Bergstrom $\mathrm{R}$, Holmberg L, Mallmin $\mathrm{H}$, et al. Excessive dietary intake of vitamin $\mathrm{A}$ is associated with reduced bone mineral density and increased risk for hip fracture. Ann Intern Med. 1998; 129:770-8.

13. Feskanich, D, Singh, V, Willett, WC, Colditz GA. Vitamin A intake and hip fractures among postmenopausal women. JAMA. 2002; 287:47-54.

14. Johanson S, Lind PM, Hakansson $H$, Oxlund $H$, Örberg J, Melhus H. Subclinical Hypervitaminosis A causes fragile bones in rats. Bone. 2002; 31(6): 685-9.

15. Reeves GP, Forrest HN, Fahey CG. AIN-93 purified diets for laboratory rodents: final report of the Americam Institute of Nutrition Ad Hoc writing committee on the reformulation of the AIN-76 A rodent diet. J Nutr. 1993; 123:1939-51.

16. Mattila PT, Svanberg, MJ, Jãmsã T, Knuuttila MLE. Improved Bone Biomechanical Properties in XylitolFed Aged Rats. Metabolism. 2002; 51(1):92-6.

17. StatSoft Inc. STATISTICA (data analysis software system) version 6.0. Tulsa: StatSoft; 2001.

18. Anderson JJB. Oversupplementation of vitamin A and osteoporotic fractures in the elderly: to supplement or not to supplement with vitamin A. J Bone Miner Res. 2002; 17:1359-62.

19. Denke MD. Dietary retinol: a double-edged sword. JAMA. 2002; 287:102-4.
20. Frame B, Jackson CE, Reynolds WA, Umphrey JE. Hypercalcemia and skeletal effects in chronic hypervitaminosis A. Ann Inter Med. 1974; 80:44-8.

21. Jowsey J, Riggs BL. Bone changes in a patient with hypervitaminosis A. J Clin Endocrinol Metab. 1968; 28:1833-5.

22. Ragavan VV, Smith JE, Bilezekian JP. Vitamin A toxicity and hipercalcemia. Am J Med Sci. 1982; 283:161-4.

23. Frankel TL, Seshadri MS, McDowall DB, Cornish CJ. Hypervitaminosis $A$ and calcium-regulating hormones in rat. J Nutr. 1986; 116:578-87.

24. Leelaprute $\vee$, Boonpucknavig $\vee$, Bhamarapravati N, Weerapradist W. Hypervitaminosis A in rats. Arch Pathol Lab Med. 1973; 96:5-9.

25. Moore T, Wang YL. Hypervitaminosis A. Biochem J.1945; 39:222-8.

26. Högström $M$, Nordström A, Nordström P. Retinol, retinol-binding protein 4 , abdominal fat mass, peak bone mineral density, and markers of bone metabolism in men: the Northern Osteoporosis and Obesity (NO2) Study. Eur J Endocrinol. 2008; 158(5):765-70.

27. Kawahara TN, Krueger DC, Engelke JA, Harke JM, Birkley NC. Short-Term Vitamin A supplementation does not affect bone turn over in men. J Nutr. 2002; 132:1169-72.

28. Li XF, Dawson-Hughes B, Hopkins R, Russell RM, Jee WS, Bankson D, et al. The effects of chronic vitamin $A$ excess on bone remodeling in aged rats. Proc Soc Exp Biol Med. 1989; 191:103-7.

29. Nieman C, Obbink $H$. The biochemistry and pathology od hipervitaminosis A. Vitam Horm. 1998; 12:69-99.

30. Hough S, Avioli LV, Muir H, Gelderblom D, Jenkins $\mathrm{G}$, Kurasi $\mathrm{H}$, et al. Effects of hypervitaminosis $A$ on the bone and mineral metabolis of the rat. Endocrinology. 1988; 122:2933-9.

Recebido em: 3/8/2009

Versão final reapresentada em: 2/12/2010 Aprovado em: 21/2/2011 
